\title{
High-throughput beamline for attosecond pulses based on toroidal mirrors with microfocusing capabilities
}

Cite as: Rev. Sci. Instrum. 85, 103115 (2014); https://doi.org/10.1063/1.4898671

Submitted: 02 July 2014 . Accepted: 08 October 2014 . Published Online: 24 October 2014

F. Frassetto, A. Trabattoni, S. Anumula, G. Sansone, F. Calegari, M. Nisoli, and L. Poletto

\section{ARTICLES YOU MAY BE INTERESTED IN}

Design and test of a broadband split-and-delay unit for attosecond XUV-XUV pump-probe experiments

Review of Scientific Instruments 87, 023106 (2016); https://doi.org/10.1063/1.4941722

Attosecond beamline with actively stabilized and spatially separated beam paths

Review of Scientific Instruments 86, 123106 (2015); https://doi.org/10.1063/1.4937623

A high-flux high-order harmonic source

Review of Scientific Instruments 84, 073103 (2013); https://doi.org/10.1063/1.4812266

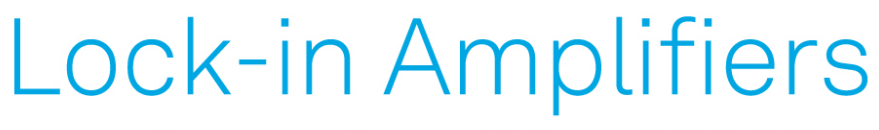

... and more, from DC to $600 \mathrm{MHz}$

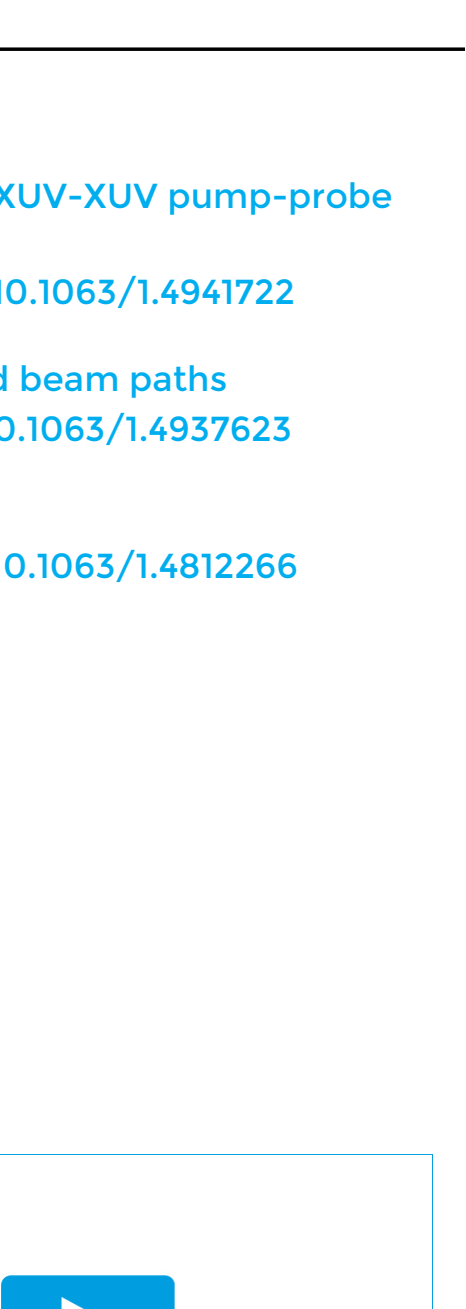




\title{
High-throughput beamline for attosecond pulses based on toroidal mirrors with microfocusing capabilities
}

\author{
F. Frassetto, ${ }^{1}$ A. Trabattoni, ${ }^{2}$ S. Anumula, ${ }^{2}$ G. Sansone,${ }^{2}$ F. Calegari, ${ }^{3}$ M. Nisoli, ${ }^{2,3}$ \\ and L. Poletto ${ }^{1, a)}$ \\ ${ }^{1}$ National Research Council, Institute of Photonics and Nanotechnologies, via Trasea 7, 35131 Padova, Italy \\ ${ }^{2}$ Department of Physics, Politecnico di Milano, Piazza L. Da Vinci 32, 20133 Milano, Italy \\ ${ }^{3}$ National Research Council, Institute of Photonics and Nanotechnologies, Piazza L. da Vinci 32, \\ 20133 Milano, Italy
}

(Received 2 July 2014; accepted 8 October 2014; published online 24 October 2014)

\begin{abstract}
We have developed a novel attosecond beamline designed for attosecond-pump/attosecond probe experiments. Microfocusing of the Extreme-ultraviolet (XUV) radiation is obtained by using a comacompensated optical configuration based on the use of three toroidal mirrors controlled by a genetic algorithm. Trains of attosecond pulses are generated with a measured peak intensity of about $3 \times 10^{11} \mathrm{~W} / \mathrm{cm}^{2}$. ( 2014 AIP Publishing LLC. [http://dx.doi.org/10.1063/1.4898671]
\end{abstract}

\section{INTRODUCTION}

The production of attosecond pulses by high-order harmonic generation (HHG) in gas is now a mature technique and several applications of attosecond pulses have been reported. ${ }^{1-4}$ In a typical attosecond measurement an attosecond pulse is used in combination with a visible/near infrared (IR) pulse with femtosecond duration. The use of this twocolor configuration is mainly related to the low photon flux of the available attosecond sources, in particular in the case of isolated attosecond pulses. In the last few years significant progress has been obtained in the generation of highflux attosecond pulses. Various techniques have been implemented as the two-color field synthesis in combination with loose-focusing geometry ${ }^{5}$ and the interferometric polarization gating scheme, ${ }^{6}$ thus opening the way to attosecondpump/attosecond-probe experiments. ${ }^{7}$

The typical power density required to investigate nonlinear processes, or processes having unfavorable cross section, is of the order of $10^{12}-10^{14} \mathrm{~W} / \mathrm{cm}^{2}{ }^{8,9}$ Two approaches can be adopted to achieve high Extreme-ultraviolet (XUV) intensity: increase of the energy of the attosecond pulse and/or decrease of the XUV spot size on target. Given the brilliance of HHG sources driven by femtosecond pulses with tens of millijoule energy, the intensity values suitable for XUV-XUV applications can be reached by focusing the XUV radiation in a spot with sub-10- $\mu \mathrm{m}$ diameter. Assuming a generation source of the order of several tens of micrometers, this can be obtained by using a single mirror (e.g., an ellipsoidal or a parabolic mirror) with the appropriate demagnification factor. Unfortunately, unless using unfavorable long entrance arm, this solution forces to use mirrors with very short exit arm, ${ }^{10}$ that have to be consequently installed inside the experimental chamber. The increase of the length of the output arm requires at least the use of two mirrors, the first providing the required demagnification factor and the second used as a relay optic. Furthermore, XUV-XUV pump-probe capabilities require an almost perfect spatial overlapping on

a) Author to whom correspondence should be addressed. Electronic mail: poletto@dei.unipd.it. the target area between the two XUV pulses, which must be maintained when the pump-probe relative delay is changed typically by moving a plane split mirror. This condition cannot be achieved when the split mirror is inserted in a converging/diverging beam, since the position of the focus would change with the delay. Consequently, the XUV beam has to be split where it is collimated. The first focusing mirror has indeed to be replaced with a couple of ideally parabolic mirrors, acting, respectively, as collimator and focusing, with the split mirror placed in between. Therefore, the whole beamline consists of four mirrors at grazing incidence, namely, collimator (paraboloid), split (plane), focusing (paraboloid), and relay (ellipsoidal). Such a geometry is able to provide: (i) high demagnification of the XUV source; (ii) long exit arm although maintaining a compact envelope, thus offering the possibility to introduce different experimental setups in the focal region for pump-probe measurements; (iii) generation of two delayed XUV pulse replica in an intermediate region where the XUV beam is collimated.

Recently, the authors presented a general configuration for an XUV-XUV beamline with the characteristics above mentioned using only toroidal mirrors. ${ }^{11}$ They are a cheaper alternative to the use of more expensive Cartesian optics, such as ellipsoids or paraboloids, with the drawback of high aberrations when used to provide high demagnification, since in this case they have to be operated highly off-Rowland. The proposed configuration is based on the use of two sections: the first one provides the required demagnification, the second one is a relay section, which provides a suitable length of the exit arm. The first section leads to the generation of a stigmatic image of the source in an intermediate focus that is free from astigmatism but has a large coma, due to the high demagnification. The relay mirror is mounted in Z-shaped geometry with respect to the previous one, so that it gives a stigmatic image with a coma that is opposite to that provided by the first section. The mirror geometrical parameters, i.e., the incidence angle and the length of its entrance and exit arms, are calculated to give a final image that is almost comafree, since the coma of the second section compensates for the coma of the first one. 
In this paper we present the application of the proposed configuration to the realization of a novel attosecond beamline designed for XUV-pump/XUV probe applications. We present the design of the beamline and the measurements of the spatial characteristics of the attosecond pulses at the output. The alignment of the toroidal mirrors of the beamline is performed through a genetic algorithm which controls the translations and rotations of the optical elements. Trains of attosecond pulses are generated with a measured peak intensity of about $5 \times 10^{11} \mathrm{~W} / \mathrm{cm}^{2}$.

\section{BEAMLINE DESIGN}

The experimental setup of the beamline, which is shown in Fig. 1(a), is based on the use of three toroidal mirrors at grazing incidence (provided by Hellma Optics, Germany) and a plane split-mirror for the generation of two pulse replica with variable temporal delay. The toroidal mirrors are mounted on 6-axis motorized vacuum actuators (provided by MICOS, Germany and Newport, USA). The mirror pair TM1TM2 is required for beam collimation and demagnification, while mirror TM3 is the relay section that is used to refocus the beam with a long exit arm. The appropriate choice of parameters and orientation of the relay mirror TM3 with respect to the focusing mirror TM2 makes it possible to almost completely cancel the coma aberration introduced by the toroidal mirrors when used with high demagnification. ${ }^{12}$

The split mirror is realized using two combined flat mirrors SM1 and SM2, as discussed in Ref. 13 and shown in Fig. 1(b). SM2 is mounted on a piezo translation stage, and is used for the time delay scan, while SM1 is kept fixed. The temporal delay between the two split pulses is $\mathrm{T}=2 d \operatorname{sen} \theta / \mathrm{c}$, where $\theta$ is the grazing angle on the mirrors and $d$ the separation between SM1 and SM2. Since in our case $\theta=10^{\circ}$, temporal resolution of 10 as is obtained with a control on $d$ of $10 \mathrm{~nm}$, that is well within the capabilities of the piezo actuator. The suppression of the IR fundamental beam is realized by inserting an aluminum foil after the split mirror.

The beamline parameters are summarized in Table I. They have been chosen to satisfy the condition for coma compensation, given by the following condition as obtained in

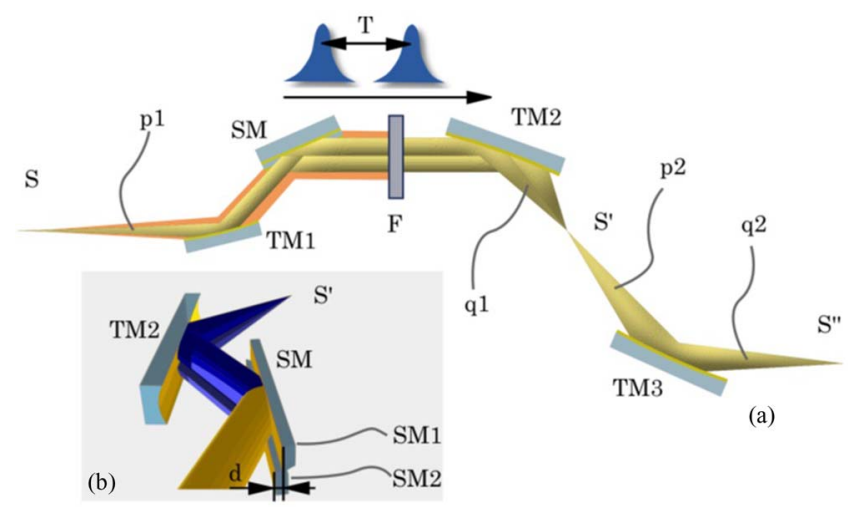

FIG. 1. Beamline design. (a) Schematics: $\mathrm{S}$ is the attosecond pulses generation point, TM1 the collimator, SM the split mirror, F the Al filter foil, TM2 the focusing mirror, $\mathrm{S}^{\prime}$ the intermediate focal spot (coma aberrated), TM3 the relay mirror, and $\mathrm{S} / /$ the output spot (coma free). (b) SM working principle.
TABLE I. Beamline parameters.

\begin{tabular}{lc}
\hline \hline Toroidal mirror TM1 & Collimator \\
\hline Entrance arm $p_{1}$ & $1500 \mathrm{~mm}$ \\
Incidence angle & $80^{\circ}$ \\
Radii (tangential $\times$ sagittal) & $17.3 \mathrm{~m} \times 520 \mathrm{~mm}$ \\
Size (tangential $\times$ sagittal) & $40 \mathrm{~mm} \times 10 \mathrm{~mm}$ \\
Coating & Gold \\
Toroidal mirror TM2 & Focusing \\
\hline Exit arm $q_{1}$ & 150 \\
Incidence angle & $80^{\circ}$ \\
Radii (tangential $\times$ sagittal) & $1.73 \mathrm{~m} \times 52 \mathrm{~mm}$ \\
Size (tangential $\times$ sagittal) & $40 \mathrm{~mm} \times 10 \mathrm{~mm}$ \\
Coating & Gold \\
Toroidal mirror TM3 & Relay \\
\hline Entrance arm $p_{2}$ & 660 \\
Exit arm $q_{2}$ & 600 \\
Incidence angle & $80^{\circ}$ \\
Radii (tangential $\times$ sagittal) & $3.62 \mathrm{~m} \times 109 \mathrm{~mm}$ \\
Size (tangential $\times$ sagittal) & $100 \mathrm{~mm} \times 25 \mathrm{~mm}$ \\
Coating & Gold \\
General parameters & \\
\hline Input angular acceptance $(\mathrm{max})$ & $\pm 2 \mathrm{mrad}$ \\
Maximum split-pulses delay & $80 \mathrm{fs}$ \\
Total demagnification & 11 \\
Total length of the beamline & $3.1 \mathrm{~m}$ \\
Beamline transport efficiency & 0.3 \\
\hline \hline & \\
\hline &
\end{tabular}

Ref. 11 from the optical aberration theory:

$$
\mathbf{M}_{2}=\left(1+p_{1} /\left(q_{2} \mathrm{M}_{1}\right)\right)^{1 / 2}
$$

where $\mathrm{M}_{1}=p_{1} / q_{1}$ and $\mathrm{M}_{2}=p_{2} / q_{2}$ and are the demagnification factors of the two sections.

\section{BEAMLINE CHARACTERIZATION}

\section{A. Coma compensation}

We have primarily verified the coma compensation capabilities, testing the beamline by using a $\mathrm{He}-\mathrm{Ne}$ laser beam transmitted through a $50 \mu \mathrm{m}$ pinhole placed in the XUV source position, $\mathrm{S}$, acting as a point-like source. The two split mirrors have been initially replaced by a single flat mirror. Two optical configurations have been tested to assess the reliability of the optical design: the scheme shown in Fig. 1(a), where TM2 and TM3 face one to each other, hereafter called compensating configuration (Z-Conf), and the non-compensating scheme (C-Conf), where the toroidal mirrors TM2 and TM3 face the same hemi-space. The results are reported in Fig. 2. While the use of the Z-Conf results in an excellent coma compensation, the C-Conf scheme leads to an amplification of coma aberration. The experimental results are predicted also by the ray-tracing modeling of the beamline, as shown in Fig. 3 .

We have also calculated by ray-tracing the spread of the optical paths at the output as resulting from the residual aberrations, which determine the ultimate temporal resolution of the optical setup. It results 100 as full-width-at-halfmaximum (FWHM) with 2 mrad beam divergence. 


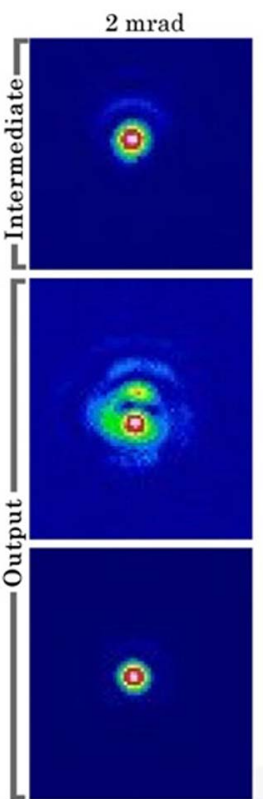

(a)
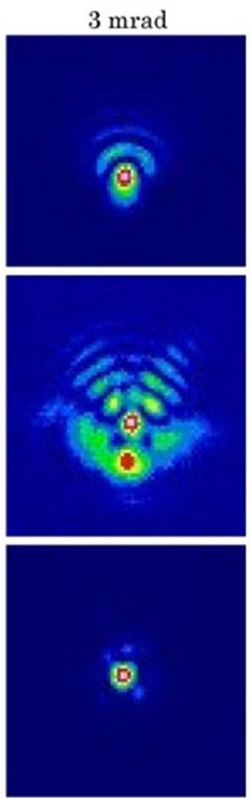

(b)
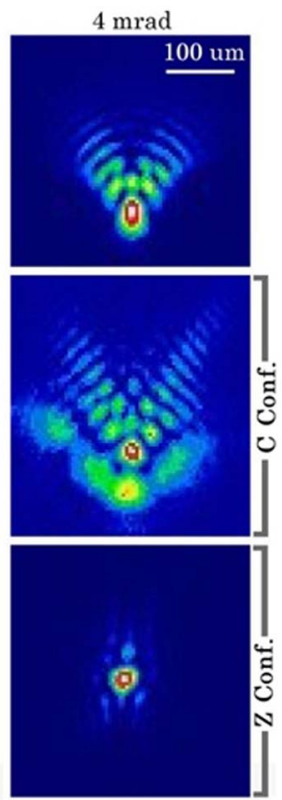

(c)
FIG. 2. (a)-(c) Focal spots measured in visible light in the intermediate $\left(S^{\prime}\right)$ and output $\left(\mathrm{S}^{\prime \prime}\right)$ planes for three beam divergences. Both C-Conf and Z-Conf have been tested.

\section{B. XUV generation and microfocusing}

The driving source is a Ti:sapphire amplified laser system which generates 25 fs pulses with an energy of $6 \mathrm{~mJ}$, repetition rate of $1 \mathrm{kHz}$ and stabilized carrier envelope phase (FEMTOLASERS - Femtopower Pro V CEP). These pulses are compressed by using the hollow fiber technique in combination with the pressure gradient scheme ${ }^{14,15}$ down to $6 \mathrm{fs}$ with an energy of $2 \mathrm{~mJ}$. Dispersion compensation has been
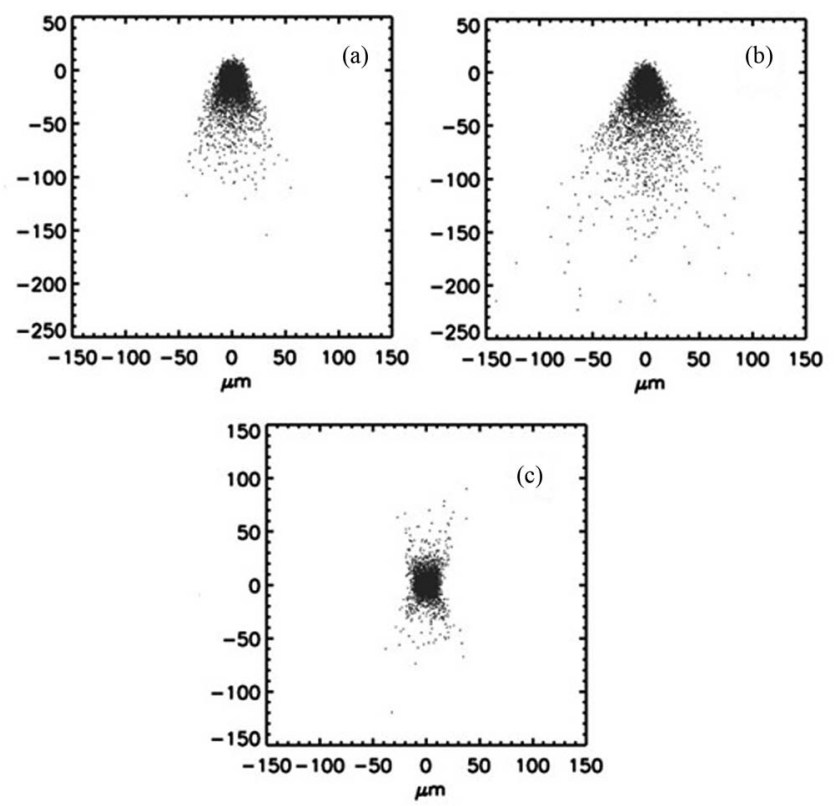

FIG. 3. Ray-tracing of the XUV beamline, $100 \mu \mathrm{m}$ FWHM source, $1.0 \mathrm{mrad}$ FWHM divergence: (a) spot in $\mathrm{S}^{\prime}$, coma-aberrated; (b) spot in $\mathrm{S}^{\prime \prime}$ in the noncompensating configuration (C-Conf), coma-aberrated; (c) spot in $\mathrm{S}^{\prime \prime}$ in the compensating configuration (Z-Conf), almost coma-free.

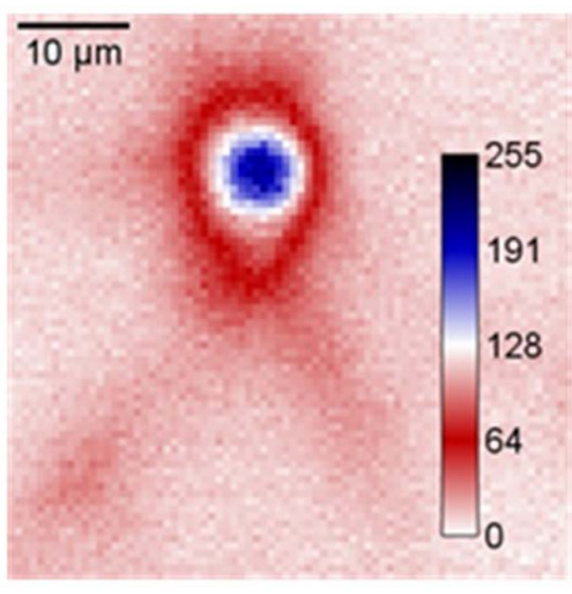

FIG. 4. Output focal spot acquired by the Ce-YAG scintillator. The FWHM diameter is $8 \mu \mathrm{m}$.

achieved by ultra-broadband chirped mirrors. XUV pulses have been produced by HHG in a 10-mm-long cell filled with krypton at static pressure. The XUV spectrum, as acquired by the flat-field spectrometer described in Ref. 16, ranges from 17 to $38 \mathrm{eV}$.

The XUV focal spot in $\mathrm{S}^{\prime \prime}$ has been measured by a 0.5-mm-thick Ce-YAG scintillator. The visible light fluorescence induced by the XUV radiation has been acquired using a microscope objective providing a $10 \times$ magnification and a CMOS camera, giving a spatial resolving element of $0.67 \mu \mathrm{m}$. The spot resulting after the fine alignment of the mirrors is shown in Fig. 4. It is almost circular with $8 \mu \mathrm{m}$ FWHM diameter, showing no evidence of coma. Given the total beamline demagnification of 11, the XUV source diameter is expected to be about $90 \mu \mathrm{m}$ FWHM diameter.

The quality of the spot size depends from the relative alignment of the three concave mirrors. The error budget, here defined as the translations and rotations of the toroidal mirrors producing a broadening of the focal spot by a factor two, is shown in Table II. Here $z$ is defined as the axis normal to the optical surface and $y$ as that along the beam propagation direction. The precision in the alignment is quite restrictive especially for TM2 and TM3. The optimization of the output focal spot is consequently a time-consuming operation.

To facilitate the day-to-day operation of the beamline, the movements of the toroidal mirrors have been controlled by implementing a genetic algorithm. ${ }^{17}$ The control has been applied on the twelve motors that move TM2 and TM3. The fitness function $\mathrm{F}$ that has been used to provide the feedback to the motors has been selected as

$$
\mathrm{F}=\mathrm{I}_{\text {max }}^{4} /\left[\mathrm{N} \times\left(\left|\mathrm{N}_{\mathrm{x}}-\mathrm{N}_{\mathrm{y}}\right|+1\right)\right],
$$

TABLE II. Beamline error budget.

\begin{tabular}{|c|c|c|c|c|c|c|}
\hline & \multicolumn{3}{|c|}{ Translation (mm) } & \multicolumn{3}{|c|}{ Rotation (deg) } \\
\hline & $\mathrm{x}$ & $\mathrm{y}$ & $\mathrm{z}$ & $\mathrm{x}$ & Y & $\mathrm{z}$ \\
\hline TM1 & 3 & $>10$ & 1 & 0.1 & 0.3 & 2 \\
\hline TM2 & 0.3 & 0.8 & 0.2 & 0.03 & 0.3 & 0.2 \\
\hline TM3 & 0.3 & 1 & 0.2 & 0.02 & 0.15 & 0.02 \\
\hline
\end{tabular}



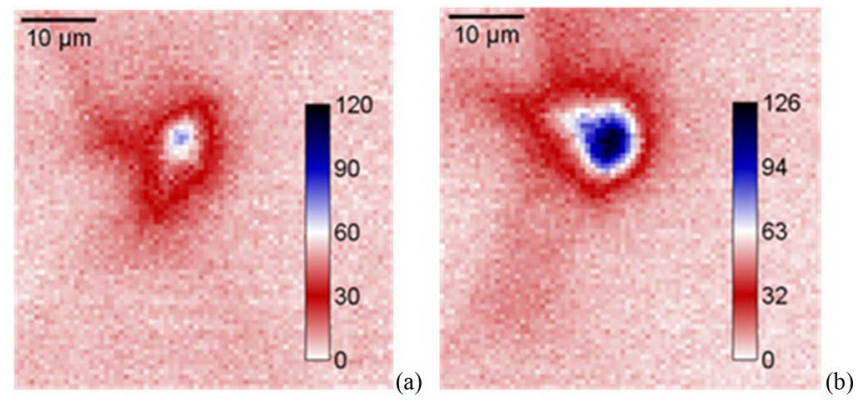

FIG. 5. Spot optimization using the genetic algorithm: (a) spot at the beginning of the optimization cycle, with the mirrors relatively misaligned; (b) spot after the alignment optimization cycle, $8 \mu \mathrm{m}$ FWHM diameter.

where $\mathrm{I}_{\max }$ is the maximum spot intensity, $\mathrm{N}$ is the number of pixels with intensity greater than $\mathrm{I}_{\max } / 2, \mathrm{~N}_{\mathrm{x}}$ and $\mathrm{N}_{\mathrm{y}}$ are the number of columns and rows, respectively, with intensity greater than $\mathrm{I}_{\max } / 2$. Aim of the function $\mathrm{F}$ is to reward the maximum spot intensity, the minimum spot size, and the spot symmetry. The result of the automatic optimization cycle is shown in Fig. 5. The quality of the final image, which has been obtained after an optimization cycle of $10 \mathrm{~min}$, is close to the optimum image obtained with the manual alignment. In the normal operational mode, a plane mirror can be inserted in the beam path just after TM3, thus providing an additional off-line image of $S^{\prime \prime}$, that will be used to close the loop for the optimization routine.

To complete the spatial characterization of the beamline, the split mirror has been inserted in the optical path. The focal spot measured by using the Ce:YAG scintillator is shown in Fig. 6, after proper alignment of SM1 and SM2. The spot FWHM diameter is $8 \mu \mathrm{m}$ also in this case, although some degradation in the image is visible compared to the case with a single plane mirror, due to diffraction and scattering from the illuminated borders of SM1 and SM2.

Despite the use of four optics, the transmission of the beamline is quite high, $\mathrm{T} \approx 0.30$ for linear s-polarization, obtained from the measurement of the reflectivity of the mirrors used in the beamline. Moreover, the efficiency is almost constant in the 30-70 nm wavelength region, due to the flat

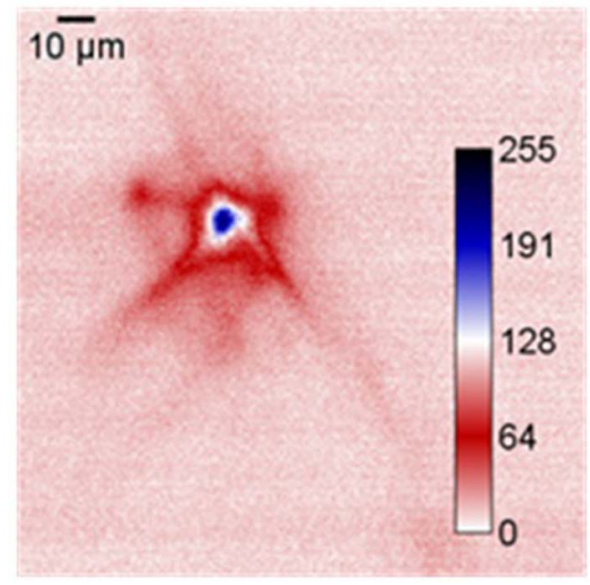

FIG. 6. Output focal spot with the split mirror inserted, SM1 and SM2 coaligned. The FWHM diameter is $8 \mu \mathrm{m}$. reflectivity curve at grazing incidence. Compared to different implementations used for microfocusing of XUV radiation, where a gold-coated mirror at normal incidence is used to split and focus the radiation (e.g., see Ref. 9), our novel configuration gives higher throughput, since gold reflectivity at normal incidence is $\approx 0.10$ in the $40-60 \mathrm{~nm}$ region.

\section{XUV intensity}

Harmonic generation driven by 6 fs pulses leads to the production of a short train of attosecond pulses separated by half optical cycle of the fundamental radiation. In order to measure the number of the most intense pulses in the train we have measured the interference fringes originated on the Ce-YAG crystal placed at the output, $\mathrm{S}^{\prime \prime}$, upon changing the temporal delay between the two pulse replica generated by the plane split mirror. ${ }^{17}$ Interference fringes appear only once, with two faint pre/post patterns when the delay is changed by $\approx 1.2$ fs (corresponding to half optical cycle of the IR driving pulse), thus indicating that in our experimental conditions the XUV energy is predominantly contained in two attosecond pulses separated by $1.2 \mathrm{fs}$. The other pulses of the train do not significantly contribute to the total energy of the XUV pulse. Moreover, the observation of stable interference fringes demonstrates the excellent mechanical stability of the optical setup.

The energy of the attosecond pulses at the output, $\mathrm{S}^{\prime \prime}$, has been measured by using a calibrated XUV photodiode: an energy value of $0.4 \mathrm{~nJ}$ has been obtained. By using the measured spot size of the XUV radiation and assuming an overall XUV pulse duration of $1.2 \mathrm{fs}$, a peak intensity of 3.3 $\times 10^{11} \mathrm{~W} / \mathrm{cm}^{2}$ can be obtained. This is a remarkable result, given the low energy of the driving pulses used in the experiments. Due to the low energy of the driving pulses $(2 \mathrm{~mJ})$ the XUV peak intensity obtained in the beamline described in this work is not high enough for XUV-pump/XUV-probe measurements. Upon using driving pulses with tens of millijoules energy it is possible to increase by orders of magnitude the XUV pulse energy preserving the same excellent spatial characteristics.

\section{CONCLUSION}

We have realized and characterized a novel attosecond beamline designed for attosecond-pump/attosecond-probe measurements. By using an optical setup based on the use of three toroidal mirrors combined to compensate for coma aberration, it is provided: (i) high demagnification of the XUV source, that is a factor 11 in the geometry here described; (ii) long exit arm in a table-top compact environment, that is $0.6 \mathrm{~m}$ for a total envelope of about $3 \mathrm{~m}$, thus offering the possibility to introduce different experimental setups in the focal region for pump-probe measurements; (iii) generation of two delayed XUV pulse replica in an intermediate region where the XUV beam is collimated, that guarantees the spatial superposition of the two split pulses when changing the relative delay. The use of toroidal mirrors is a much cheaper solution with respect to Cartesian surfaces for experiments requiring a 
temporal resolution of few hundreds of attoseconds. Finally, an XUV spot with no evidence of coma and FWHM diameter of $8 \mu \mathrm{m}$ has been measured.

The configuration here proposed may be extended to a further decrease the focal spot down to very few microns. Assuming the same parameters for the source as those measured in this experimental set-up, it can be shown by ray-tracing simulations that a couple of toroidal mirrors can be chosen to give a total demagnification of about 26 with an entrance arm of $2000 \mathrm{~mm}$, a final exit arm of $500 \mathrm{~mm}$, and a total length of about $3100 \mathrm{~mm}$. The final spot size is expected to be $3.5 \mu \mathrm{m}$ FWHM diameter, as confirmed also by ray-tracing simulations. As a general indication, for spot sizes in the very few micron level, although the coma aberration may be almost perfectly cancelled as analytically calculated, the residual higher-order aberrations, mainly the spherical aberration, have to be taken into account and may limit the final size of the output spot. The mirror parameters, as calculated from Eq. (1), have to be anyway confirmed by ray-tracing simulations, that are used to find the best trade-off for the compensation of the image aberrations. For a further decrease of the spot size in the sub-micron level, the main limit to the use of toroidal mirrors is given by the residual surface slope errors that may irreparably degrade the final quality of the spot. Indeed, in case of very high demagnifications the configuration has to be designed taking into account the actual parameters on the surface quality that are provided by the vendor.

\section{ACKNOWLEDGMENTS}

This work was supported by the European Research Council under the European Community's Seventh Frame- work Programme (FP7/2007-2013)/ERC Grant agreement No. 227355-ELYCHE, by LASERLAB-EUROPE (Grant agreement No. 284464, EC's Seventh Framework Programme) and by the Italian Ministry of Research (Project. FIRB No. RBID08CRXK).

${ }^{1}$ F. Krausz and M. Ivanov, Rev. Mod. Phys. 81, 163-234 (2009).

${ }^{2}$ G. Sansone, L. Poletto, and M. Nisoli, Nat. Photon. 5, 655-663 (2011).

${ }^{3}$ M. Chini, K. Zhao, and Z. Chan, Nat. Photon. 8, 178-186 (2014).

${ }^{4}$ F. Lépine, M. Y. Ivanov, and M. J. J. Vrakking, Nat. Photon. 8, 195-204 (2014).

${ }^{5}$ E. J. Takahashi, P. Lan, O. D. Mücke, Y. Nabekawa, and K. Midorikawa, Nat. Commun. 4, 2691 (2013).

${ }^{6}$ P. Tzallas, E. Skantzakis, C. Kalpouzos, E. P. Benis, G. D. Tsakiris, and D. Charalambidis, Nat. Phys. 3, 846-850 (2007).

${ }^{7}$ P. A. Carpeggiani, P. Tzallas, A. Palacios, D. Gray, F. Martin, and D. Charalambidis, Phys. Rev. A 89, 023420 (2014).

${ }^{8}$ Y. H. Jiang, A. Rudenko, O. Herrwerth, L. Foucar, M. Kurka, K. U. Kühnel, M. Lezius, M. F. Kling, J. van Tilborg, A. Belkacem, K. Ueda, S. Düsterer, R. Treusch, C. D. Schröter, R. Moshammer, and J. Ullrich, Phys. Rev. Lett. 105, 263002 (2010).

${ }^{9}$ P. Tzallas, E. Skantzakis, L. A. A. Nikolopoulos, G. D. Tsakiris, and D. Charalambidis, Nat. Phys. 7, 781-784 (2011).

${ }^{10}$ H. Mashiko, A. Suda, and K. Midorikawa, Opt. Lett. 29(16), 1927-1929 (2004).

${ }^{11}$ L. Poletto, F. Frassetto, F. Calegari, S. Anumula, A. Trabattoni, and M. Nisoli, Opt. Express 21, 13040-13051 (2013).

${ }^{12}$ L. Poletto, P. Nicolosi, and G. Tondello, Appl. Opt. 41, 172-181 (2002).

${ }^{13}$ T. Shimizu, T. Okino, K. Furusawa, H. Hasegawa, Y. Nabekawa, K. Yamanouchi, and K. Midorikawa, Phys. Rev. A 75, 033817 (2007).

${ }^{14}$ M. Nisoli, S. De Silvestri, and O. Svelto, Appl. Phys. Lett. 68, 2793 (1996).

${ }^{15}$ A. Suda, M. Hatayama, K. Nagasaka, and K. Midorikawa, Appl. Phys. Lett. 86, 111116 (2005)

${ }^{16}$ L. Poletto, S. Bonora, M. Pascolini, and P. Villoresi, Rev. Sci. Instrum. 75, 4413-4418 (2004).

${ }^{17}$ P. Villoresi, S. Bonora, M. Pascolini, L. Poletto, G. Tondello, C. Vozzi, M. Nisoli, G. Sansone, S. Stagira, and S. De Silvestri, Opt. Lett. 29, 207-209 (2004). 